\title{
CRISIS DECISION MAKING AND MANAGEMENT
}

Crisis management studies is a research area that analyzes how public and private actors respond to events that threatens individuals and society. There are many organizational and societal processes that enter this empirical space and several social science disciplines have contributed to the development of this research area, such as sociology, political science, psychology, public administration, and international relations to name some of the most prominent. A core question when studying the management of crises will always be: what characterizes high stake decisions made under pressing circumstances? When starting to answer this question, we need an understanding of both psychological and institutional forces that surround the situation in order to see the individual decision-maker in context.

\section{Overview}

Societal crises, according to a well-established definition, are characterized by a threat to core societal values, time pressure, and uncertainty. The concurrence of these factors, each a potentially destabilizing influence on situational awareness and social interaction, constitutes a unique combination of organizational and psychological challenges for arriving at collective decisions. Individuals experience a heightened level of stress, typically causing an effect on performance that describes an inverted U-curve. In modest amounts, psychological stress increases performance of mental tasks but over a certain level, decision-makers experience an impaired ability to evaluate the circumstances and 
deal with complexity. Decision-making under stress is hampered by deterioration in information processing, the incorporation of fewer tactical and strategic options, and a restriction in the number of individuals involved in the decision-making process. For all the mental and organizational limitations introduced by stress, some benefits can also be identified in the literature: more attention is often devoted to the problem at hand; resources can be redistributed to deal with it; and allies can be summoned to contribute to managing the situation.

An increasingly central way of categorizing human decision-making is to differentiate between the cognitive systems 1 and 2 . System 1 consists of the instinctive identification of a dominant option based on a cursory scan of previous experiences. This is how numerous everyday decisions are made and their instinctive quality makes them virtually effortless. The problem is that if the potential consequences are serious, system 1 does not help us assess the complexity of the decision or its risk structure. In the more consequential situation, decision theorists claim that we are better served by system 2 , which has a more formalized approach to the problem. The decision-maker assess the relative satisfaction to be gained from each outcome, and the likelihood that it will come about. System 1 and 2 are useful ways to describe the processes of choice that is pursued by individuals as well as collectives of individuals.

\section{Theories of Decision}

The classical way to look at decision-making, whether in a crisis or any other context is to take the view of the rational actors. The decision then becomes a strategic choice based 
on the utility and likelihood of different possible outcomes, a distilled version of what was previously described as system 2 . A rational actor methodically picks the option with the highest expected utility. This school of though is still dominant in a large number of social science disciplines, including economics where it once originated. Even though many of its proponents acknowledge that it does not perfectly describe every instance of individual choice, it arguably captures consequential choices on average, and is thus the best approximation possible.

The critique of the rational actor approach are multifold, but will here be represented by two primary principled objections: One says that the risk neutrality in human decisionmaking assumed by rational choice theory has been disproven. When weighting a medium to large likelihood against a dismally small one, most people overestimate the latter. The other objection comes from the observation that individuals evaluate the relative losses and gains to be had from an alternative, rather than the value of the exact outcome. This effect is known as the framing effect on choice and has certain repercussions for crisis decision-making: Crises often pose decision occasion where the stakes are high (implying a need for system 2) and the sense of possible losses are high (creating a framing effect on decision-makers). To many crisis management scholars this creates the need for a more process oriented theory of choice that probes the psychological context for the decision-makers and the resulting pressures on risk perceptions.

\section{Group decision-making}


A decision-making unit that is common in sensitive political situations is the small group. Due to needs for discretion, issues of trust, and time pressures it is common that leaders gather a small group of principals or advisers to prepare and make decisions. Social psychology is rich on the specific characteristics that can arise under the social pressure of group dynamics and crisis management studies have taken notice. Many seminal events in recent world history have been analyzed through the perspective of group decision-making. When this mode of analysis first became prominent in the 1960s, the detrimental effects of group dynamic on decision were emphasized. Groups, many argued, can easily be isolated from the larger organizational setting, be subject to pressure from the outside and develop unsound procedures for assessing risk and reaching internal consensus, in the worst case leading to a pathological phenomenon called groupthink. More recently, the view on group decision-making has become more nuanced. The social meaning of groups for their members, however, is given analytical power and developmental phases of relationships in a government can be observed over time and give clues as to the influence of certain individuals or the predominance of certain strategic alternatives.

\section{FURTHER READINGS}

Boin, R.A., 't Hart, P., Stern, E. \& Sundelius, B. (2005). The Politics of Crisis Management: Public Leadership Under Pressure. Cambridge: Cambridge University Press.

Tversky, A. \& Kahneman, D. (1981). "The framing of decisions and the psychology of choice," Science 211(4481): 453-458. 
Vertzberger, Y.Y.I. (1994). The World in Their Minds: Information Processing, Cognition, and Perception in Foreign Policy Decisionmaking. Stanford, Ca: Stanford University Press.

Zsambok, C. E., \& Klein, G. (Eds.). (2014). Naturalistic decision making. Psychology Press, London: Routledge. 